\title{
A guideline for the use of variable rate intravenous insulin infusion (VRIII) in medical inpatients
}

\author{
STELLA GEORGE, ${ }^{1}$ JANE DALE, ${ }^{2}$ DEBBIE STANISSTREET, ${ }^{1}$ ON BEHALF OF THE JOINT BRITISH DIABETES \\ SOCIETIES FOR INPATIENT CARE3 AND THE JOINT BRITISH DIABETES SOCIETIES MEDICAL VRIII \\ WRITING GROUP. ${ }^{3}$
}

\begin{abstract}
A variable rate intravenous insulin infusion (VRIII) is used commonly to achieve normoglycaemia in hospital inpatients. Most acute trusts in the UK have VRIII guidelines, but there is wide variation in the indications for its use, in rates of infusion, and in duration of use. This heterogeneity increases the risk of errors which can potentially lead to significant morbidity and mortality and also hinders study of the efficacy, optimisation and safety of VRIII. Thus, VRIII is often used when not indicated, for too long, and with inappropriate transfer to other glucose lowering medication. This article summarises the key recommendations in a recent guideline produced by the Joint British Diabetes Societies for Inpatient Care on the use of VRIII in 'medical' inpatients. The guideline is designed to be a practical guide to support the safe and effective use of VRIII by any healthcare professional who manages 'medical' inpatients with hyperglycaemia. Use of the guideline will help to harmonise the use of VRIII, with added benefits of facilitating collection of outcomes data from at multiple sites and allowing continual refinement in the therapeutic use of VRIII.

Br J Diabetes Vasc Dis 2015;15:82-85
\end{abstract}

Key words: variable rate intravenous insulin infusion, medical Inpatient, sliding scale

Rationale for the guideline

Most studies on the use of intravenous insulin infusions in hos-

Department of Diabetes and Endocrinology, East and North Herts NHS Trust, Lister Hospital, Stevenage, UK

Dudley Group NHS Foundation Trust, Dudley, UK

For full membership see the Appendix at the end of the paper

Address for correspondence: Dr Stella George

Consultant in Diabetes and Endocrinology, Department of Diabetes and

Endocrinology, East and North Herts NHS Trust, Lister Hospital,

Stevenage, SG1 4AB, UK

Tel: +44 (0)1438 284090

E-mail: stellageorge@nhs.net

http://dx.doi.org/10.15277/bjdvd.2015.014

\author{
Abbreviations and acronyms \\ CBG capillary blood glucose \\ CSII continuous subcutaneous insulin infusion \\ DKA diabetic ketoacidosis \\ HHS hyperosmolar hyperglycaemic state \\ ITU intensive therapy unit \\ JBDS-IP Joint British Diabetes Societies for Inpatient Care \\ $\mathrm{KCl}$ potassium chloride \\ $\mathrm{NaCl}$ sodium chloride \\ TDD total daily insulin dose \\ TPN total parenteral nutrition \\ VRIII variable rate intravenous insulin infusion
}

pital have been in the critical care setting or in patients who have undergone surgery. Limited data are available on the management of 'medical' inpatients (e.g. those not undergoing surgery, or those with DKA, HHS, or hyperglycaemia secondary to causes such as steroid use or enteral feeding). Local and national audits have shown that VRIII (previously known as 'sliding scale' insulin) is frequently used in hospitals to manage hyperglycaemia, but often when not indicated, for too long, and with inappropriately managed subsequent transfer to other antidiabetic therapy. ${ }^{1}$

The new guideline summarised here ${ }^{2}$ is, where possible, concordant with existing guidance from JBDS-IP in these areas, with recommendations based on extrapolation from other relevant patient groups, relevant existing JBDS guidelines, the NHS Diabetes e-learning module, 'Safe use of intravenous insulin infusion', ${ }^{3}$ and expert consensus. It is designed specifically for treating acutely unwell patients, including those with a preexisting diagnosis of diabetes and those presenting with hyperglycaemia for the first time. The appendices in the main guideline can be used as standalone summary documents for straightforward adaptation and use on the wards.

\section{General principles}

Using VRIII in hospitals

Existing guidelines (and not this one) should be used for patients with DKA, ${ }^{4} \mathrm{HHS}$ (see the new JBDS-IP guideline ${ }^{5}$ ), stroke ${ }^{6}$ and those in the perioperative period. ${ }^{7}$ Effective use of VRIII will depend on close monitoring and decision making by health care professionals, until reliable closed-loop systems become available. Incorrect use of VRIII can result in hypoglycaemia, rebound hyperglycaemia, ex- 
cess length of stay and even DKA; a safe and effective step down to other agents should occur as soon as possible.

Diabetes specialists

Refer all patients to local diabetes teams as soon as possible for assessment of individual patients' needs, to ensure that VRIII is used safely and that plans are in place for glycaemic control upon cessation of the VRIII. We recommend that all healthcare professionals complete the e-learning module on the use of intravenous insulin, ${ }^{3}$ or equivalent training.

\section{Indications for VRIII in medical inpatients}

Most acutely unwell medical patients can be managed without a VRIII, including patients who are eating and drinking normally (the diabetes team may be able to adjust these patients' insulin). A VRIII is indicated for patients with diabetes or hospital-related hyperglycaemia who are unable to take oral food/fluid and for whom adjustment of their own insulin regime is not possible, where the patient is vomiting, nil by mouth (and missing more than one meal) or severely ill with the need to achieve good control (e.g. with sepsis).

\section{Targets and threshold for starting a VRIII}

Chapter 2 of the guideline provides an extensive review of the available evidence in this area and includes discussion of glycaemic control in the settings of intensive or non-ITU care, or for patients with major adverse cardiovascular events (myocardial infarction or stroke). Guidelines from national and international societies are also discussed.

The consensus view, based on limited evidence, recommends initiation of VRIII if CBG is $>10.0 \mathrm{mmol} / \mathrm{L}$. Target blood glucose levels have not been established in trials but there is a consensus for a range between $6.0-10.0 \mathrm{mmol} / \mathrm{L}$, which should avoid risks associated with hyperglycaemia and hypoglycaemia. However, a range of $4.0-12.0 \mathrm{mmol} / \mathrm{L}$ is sometimes acceptable.

\section{Safe use of a VRIII}

In general, basal insulin should be continued, as this may help control blood glucose during the use of the VRIII and avoid rebound hyperglycaemia; all other diabetes medications should be discontinued during VRIII. Review the VRIII frequently to ensure target glucose levels are achieved, addressing hypoglycaemia promptly and adequately (according to the appropriate JBDS guideline; ; restart VRIII within 20 min of treating a hypoglycaemic episode to prevent rebound hyperglycaemia and possible ketosis). Contact the diabetes team for advice if there is doubt about how to manage hyperglycaemia, or if the blood glucose is not responding to treatment.

Monitoring during the VRIII should include hourly CBG measurements and regular review of insulin infusion rates to achieve glucose levels within the target range. Each day should include review of the continued need for VRIII, the clinical status of the patient (including fluid balance) and urea and electrolyte levels (with appropriate actions taken).

\section{Suggested insulin infusion rates}

Three rates are recommended depending on patients' likely insulin sensitivity or previous total daily dose of insulin (Table 1). Patients may move between scales depending on their response to treatment. For patients persistently out of range, first ensure proper functioning of the cannula and infusion equipment, and that the substrate infusion is running at the correct rate.

Then, if CBG remains $>12 \mathrm{mmol} / \mathrm{L}$ and is not falling on at least two consecutive measurements, the insulin infusion rate can be increased to the next scale (if the patient is already on an increased scale, employ a customised infusion and seek immediate advice from the diabetes team). Conversely, CBG 4-6 $\mathrm{mmol} / \mathrm{L}$ (on at least two consecutive measurements) may be too low for some patients (e.g. after a stroke or acute coronary syndrome). Options here are to decrease the insulin infusion rate

Table 1 Suggested scales for insulin infusion rates during a variable rate intravenous insulin infusion (VRIII)

Glucose $\mathrm{mmol} / \mathrm{L}$

Insulin rates $(\mathrm{mL} / \mathrm{h})$

Start on standard rate unless otherwise indicated

Reduced rate
(for use in insulin sensitive
patients, e.g. $\leq 24$ units/day)

Reduced rate

patients, e.g. $\leq 24$ units/day)

\section{Standard rate \\ (first choice in most patients)}

\section{Increased rate \\ (for insulin resistant patients, e.g. $\geq 100$ units per day)}

N.B. if a patient is on basal subcutaneous insulin - continue this alongside the VRIII

$<4.0$

4.1-8.0

8.1-12.0

12.1-16.0

$16.1-20.0$

20.1-24.0

$>24.1$
0 *

0.5

1

3

4
0 *

1

2

5

6
0*

2

4

6

7

8

10

See text and the full guideline for recommendations on the use of customised scales, where necessary, for defined groups of patients.

*Treat Hypoglycaemia and once CBG is $>4.0 \mathrm{mmol} / \mathrm{L}$ restart intravenous insulin within 20 minutes. The half life of intravenous insulin is very short (7-8 minutes) and restarting the VRIII promptly minimises the risk of ketosis. 
Table 2. Blood ketone results and actions to be taken

\begin{tabular}{|c|c|}
\hline Blood ketones (mmol/L) & Action \\
\hline$<0.6$ & Normal. \\
\hline $0.6-1.4$ & Check ketones in $2 \mathrm{~h}$. \\
\hline $1.5-2.9$ & $\begin{array}{l}\text { Risk of DKA } \\
\text { - Review patient management, } \\
\text { consider reasons for high ketones, } \\
\text { e.g. missed insulin dose, inadequate } \\
\text { insulin dose and address these. } \\
\text { - Call the diabetes team if in any } \\
\text { doubt. } \\
\text { - Check ketones in } 1 \mathrm{hr} \text { and take } \\
\text { action depending on the result. }\end{array}$ \\
\hline$>3.0$ & $\begin{array}{l}\text { Check venous bicarbonate or venous } \\
\mathrm{pH} \text {, change to DKA guidelines if } \\
\text { required, and inform the diabetes team. }\end{array}$ \\
\hline
\end{tabular}

or, if this has been done, to increase the substrate to $10 \%$ glucose or to prescribe a customised insulin infusion rate and promptly seek advice from the diabetes team (including to ensure that a VRIII is needed).

\section{Interpreting blood ketones during a VRIII}

Measure capillary blood ketones in patients with type 1 diabetes who are unwell or who develop persistent hyperglycaemia whilst in hospital (two readings $>12 \mathrm{mmol} / \mathrm{L}$ at least one hour apart) and in patients with type 2 diabetes who are acutely unwell (see Table 2). Urinary ketones may be used where blood ketone testing is unavailable; check venous blood bicarbonate and $\mathrm{pH}$ if urine ketones are $\geq 2+$. If venous bicarbonate is $<15 \mathrm{mmol} / \mathrm{L}$ and/or venous $\mathrm{pH}$ is $<7.3$, the patient has DKA and should be managed according to the appropriate guideline. ${ }^{4}$

\section{Management of intravenous fluids during VRIII}

Recommendations made are in line with the JBDS guideline 'Management of adults with diabetes undergoing surgery and elective procedures'.7 Monitor electrolytes daily and use fluids containing sodium if VRIII is used for $>24 \mathrm{~h}$ to avoid hyponatraemia.

We recommend the use of a balanced electrolyte solution containing $0.45 \% \mathrm{NaCl}$ with $5 \%$ glucose and $0.15 \% \mathrm{KCl}(20$ $\mathrm{mmol} / \mathrm{L})$ or $0.3 \% \mathrm{KCl}(40 \mathrm{mmol} / \mathrm{L})$, depending on potassium requirement, at $125 \mathrm{~mL} / \mathrm{h}$ in euvolaemic patients. However, as these fluids are not widely available, acceptable alternatives are:

- $5 \%$ glucose with $20 \mathrm{mmol} / \mathrm{L}$ or $40 \mathrm{mmol} / \mathrm{L} \mathrm{KCl}$ or

- $0.18 \% \mathrm{NaCl}$ with $4 \%$ glucose with $0.15 \% \mathrm{KCl}(20 \mathrm{mmol} / \mathrm{L})$ or $0.3 \% \mathrm{KCl}(40 \mathrm{mmol} / \mathrm{L})$

Patients who are vomiting, pyrexial or dehydrated will need additional fluid, usually $0.9 \% \mathrm{NaCl}$, run alongside the fluid used as the glucose substrate. Consider using 10\% glucose for patients with heart failure or severe renal or hepatic impairment to minimise risk of fluid overload.
Box 1. Calculating initial total insulin dosage (TDD) when starting an insulin regime in an insulin naïve patient or when returning an adult patient to usual insulin regime if control was previously suboptimal

\section{Weight-based calculation:}

- Frail elderly patients, CKD stage 4 or 5, new type 1 diabetes: TDD $=0.3 \times$ body weight $(\mathrm{kg})$

- All others:

TDD $=0.5 \mathrm{x}$ body weight $(\mathrm{kg})$

Estimated from the last 6 hours of the variable-rate i.v. insulin infusion (VRIII):

- Divide the total dose of insulin administered in last $6 \mathrm{~h}$ of the VRIII by 6 to calculate average hourly dose of insulin.

- Multiply this by $20^{a}$ to estimate the patient's TDD (a further correction may be needed in some patients, depending on individual insulin sensitivity, previous degree of glycaemic control and severity of intercurrent illness).

aNot by 24 , to reduce the risk of hypoglycaemia. CKD: chronic kidney disease

\section{Safe transfer to usual diabetes medication}

Return to usual antidiabetic therapy will only be appropriate when CBG targets on VRIII have been achieved and the patient has recovered from the precipitating illness/condition and is eating and drinking reliably. Background insulin should have been continued, but if not, or if the patient is insulin-naïve, background insulin (mixed insulin or within a basal bolus regime) MUST be given prior to stopping the VRIII. The VRIII should only be discontinued 30 minutes after subcutaneous insulin has been given, ideally be at meal time, after administration of shortacting or mixed insulin (see below for details of how to identify an appropriate initial dosage of insulin). Avoid stopping VRIII at bedtime where there is less observation by staff. If CBG rises after the VRIII is discontinued, contact the diabetes team do not restart the infusion. For non-insulin treated diabetes patients, give the usual treatment prior to discontinuing VRIII and seek advice from the diabetes team if control prior to admission was suboptimal.

Patients new to insulin need education and support in diabetes self-management and must be confident in self-injecting prior to discharge. Continued support must be available from appropriately-trained professionals. For adjusting an existing insulin regimen to address sub-optimal glycaemic control, use of a weight-based calculation or a dose based on insulin requirements during the stable phase of the VRIII are equally appropriate (see Box 1). Calculate the TDD using either method and use this to convert the patient to either a twice daily premix regimen (usually $60 \% / 40 \%$ of daily insulin given with breakfast/evening meal) or basal bolus regimen (usually $50 \%$ of daily insulin as basal, with the remainder as prandial insulin in equivalent doses after breakfast, lunch and evening meal).

Review the response to the new insulin regimen before 


\section{Key messages}

- Variability between UK centres in indications for the use of variable rate intravenous insulin infusions (VRIII), with frequent inappropriate initiation or withdrawal of this intervention, increases the risk of significant additional morbidity and mortality in inpatients with diabetes

- The Joint British Diabetes Societies for Inpatient Care have produced a new guideline for the use of VRIII

- The new guideline, summarised here, is based on clinical evidence (where available), extrapolation from other relevant JBDS guidelines, and expert consensus

- It is intended to support the practice of any healthcare professional caring for medical inpatients with diabetes

- Harmonising the use of VRIII will facilitate both the collection data on clinical outcomes in these patients and the future refinement of this technique

discharge, checking CBG one hour after discontinuing VRIII, at least four-hourly for the first $24 \mathrm{~h}$ after VRIII has been discontinued, and 2-4 times daily for all inpatients on subcutaneous insulin. Blood glucose $6-10 \mathrm{mmol} / \mathrm{L}$ is usually appropriate, with 4-12 mmol/L appropriate for some with significant comorbidities. All patients/carers must be confident in using the insulin delivery device prior to resuming self-management.

\section{Special circumstances}

Recommendations are also provided for the use of a VRIII in special circumstances such as during the use of TPN or CSII.

\section{Conclusions}

The JBDS-IP writing group has produced a document designed to guide non-specialists in the safe use of VRIII, for use in acutely unwell medical inpatients. It has been designed so that trusts can adapt it for use locally and we would welcome feedback to the authors to inform future revisions.

Acknowledgements Special thanks to Christine Jones for her administrative work and help with these guidelines and with JBDS-IP.

\section{Conflict of interest None.}

Funding No funds were received directly by the authors though travel expenses were met for all the writing group by the JBDS as received from DUK and $A B C D$.

\section{Appendix: Contributors to the guideline}

\section{The JBDS-IP Group:}

Belinda Allan; Hamish Courtney; Ketan Dhatariya; Daniel Flanagan; Stella George; Rob Gregory; June James; Tracy Kelly; Rif Malik; Colin Perry; Gerry Rayman; Stuart Ritchie; Aled Roberts; Mike Sampson; Maggie Sinclair-Hammersley; Debbie Stanisstreet; Jonathan Valabhji; Esther Walden; Peter Winocour.

\section{The JBDS-IP Writing Group}

Ali Abbara; Linda Balian; Mithun Bhartia; Alison Cox; Frances Coyle; Anne Currie; Ketan Dhatariya; Philip Dyer; Haleema Hayat; Anne Kilvert; Abbi Lulsegged; Samson Oyibo; Tobi Parkin.

See the full guideline ${ }^{2}$ for titles and affiliations.

\section{References}

1. Health and Social Care Information Centre. National Diabetes Inpatient Audit (NaDIA) - 2012. Available at http://www.hscic.gov.uk/ catalogue/PUB10506 (accessed March 2015).

2. Joint British Diabetes Societies for Inpatient Care. The use of variable rate intravenous insulin infusion (VRIII) in medical inpatients. October 2014. Available at http://www.diabetologists-abcd.org.uk/JBDS/JBDS_IP_VRIII. pdf (accessed March 2015).

3. Virtual College. The Safe Use of Intravenous Insulin Infusion in Adults. Available at http://www.healthcareea.co.uk/the_safe_use_of_intravenous_insulin_infusion_in_adults (accessed March 2015)

4. Savage MW, Dhatariya KK, Kilvert A, et al, for the Joint British Diabetes Societies. Joint British Diabetes Societies guideline for the management of diabetic ketoacidosis. Diabet Med 2011;28:508-15. http://dx.doi.org/10.1111/j.1464-5491.2011.03246.x

5. Joint British Diabetes Societies Inpatient Care Group. The management of the hyperosmolar hyperglycaemic state (HHS) in adults with diabetes. Available at www.diabetologists-abcd.org.uk/JBDS/JBDS_IP_HHS_Adults. pdf (accessed March 2015).

6. Joint British Diabetes Societies (JBDS) for inpatient care. Glycaemic management during the inpatient enteral feeding of stroke patients with diabetes. Available at http://www.diabetologists-abcd.org.uk/JBDS/ JBDS_IP_Enteral_Feeding_Stroke.pdf (accessed March 2015).

7. Joint British Diabetes Societies. Management of adults with diabetes undergoing surgery and elective procedures: improving standards. April 2011. Available at http://www. diabetologists-abcd.org.uk/JBDS/JBDS IP_Surgery_Adults_Full.pdf (accessed March 2015).

8. NHS Diabetes/Joint British Diabetes Societies. The hospital management of hypoglycaemia in adults with diabetes mellitus. Available at www. diabetologists-abcd.org.uk/JBDS/JBDS_IP_Hypo_Adults.pdf (accessed March 2015). 